\title{
Divisive Normalization Predicts Adaptation-Induced Response Changes in Macaque Inferior Temporal Cortex
}

\author{
(1)Dzmitry A. Kaliukhovich and Rufin Vogels \\ Laboratorium voor Neuro-en Psychofysiologie, KU Leuven Medical School, 3000 Leuven, Belgium
}

Stimulus repetition alters neural responses to the repeated stimulus. This so-called adaptation phenomenon has been commonly observed at multiple spatial and temporal scales and in different brain areas, and has been hypothesized to affect the neural representation of the sensory input. Yet, the neural mechanisms underlying adaptation still remain unclear, especially in higher-order cortical areas. Here we employ a divisive normalization model of neural responses to predict adaptation-induced changes in responses of single neurons in the macaque inferior temporal (IT) cortex. According to this model, the response of a neuron is determined by an interplay between its direct excitatory and divisive normalizing inputs, with each input being subject to adaptation. To test the model, we recorded the responses of single IT cortex neurons to complex visual stimuli while separately adapting the two putative types of input to those neurons. We compared the changes in responses of these neurons following such adaptation with predictions derived from the divisive normalization model. As predicted by the model, we show that adaptation in the IT cortex can, depending on the relative strength of each putative type of input to a neuron, suppress or enhance the neural response to a complex stimulus. More generally, our data suggest that adaptation serves to selectively enhance processing of the stimuli that differ from recently experienced ones, even when these occur within a configuration of multiple stimuli.

Key words: adaptation; divisive normalization; inferior temporal cortex; macaques

Significance Statement

Stimulus repetition alters neural responses to the repeated stimulus. This so-called adaptation phenomenon has been robustly demonstrated in brains of different species and is considered to be a form of short-term plasticity inherent to the processing of sensory stimuli. Nevertheless, the functional role and underlying mechanisms of adaptation remain unclear. Here we demonstrate that divisive normalization, a canonical neural computation operating throughout the brain, predicts the adaptation-induced changes in response of single neurons to complex stimulus configurations in the macaque inferotemporal cortex. Our findings embed adaptation effects of inferotemporal neurons into the context of a broader neural network perspective that includes divisive normalization. Additionally, our findings have implications for understanding of the function of adaptation in higherorder sensory cortices.

\section{Introduction}

Adaptation effects are ubiquitous in neurons of the macaque inferior temporal (IT) cortex, the end stage of the ventral visual

Received May 26, 2015; revised April 27, 2016; accepted April 27, 2016.

Author contributions: D.A.K. and R.V. designed research; D.A.K. performed research; D.A.K. contributed unpublished reagents/analytic tools; D.A.K. analyzed data; D.A.K. and R.V. wrote the paper.

This work was supported by Fonds voor Wetenschappelijk Onderzoek Vlaanderen (G.0582.12N and G.00007.12Odysseus), Interuniversitaire Attractiepool, and Programma Financiering (PF 10/008). D.A.K. was supported by a postdoctoral fellowship of the Fonds voor Wetenschappelijk Onderzoek Vlaanderen. We thank P. Kayenbergh, G. Meulemans, I. Puttemans, C. Ulens, M. De Paep, W. Depuydt, and S. Verstraeten for technical assistance.

The authors declare no competing financial interests.

Correspondence should be addressed to Rufin Vogels, Laboratorium voor Neuro- en Psychofysiologie, KU Leuven Medical School, Campus Gasthuisberg, 0\&N2, Herestraat 49, bus 1021, 3000 Leuven, Belgium. E-mail: Rufin.Vogels@med.kuleuven.be.

DOI:10.1523/JNEUROSCI.2011-15.2016

Copyright $\odot 2016$ the authors $\quad 0270-6474 / 16 / 366116-13 \$ 15.00 / 0$ pathway. Indeed, it has been well documented that responses of IT cortex neurons usually decrease with stimulus repetition (Miller et al., 1991; Vogels et al., 1995; Sawamura et al., 2006; McMahon and Olson, 2007; Liu et al., 2009; De Baene and Vogels, 2010; Kaliukhovich and Vogels, 2011, 2012, 2014). This repetition suppression (Desimone, 1996) has aroused interest due to the widespread use of adaptation paradigms in human functional magnetic resonance imaging (fMRI) studies (Malach, 2012). Thus, a better understanding of adaptation mechanisms in the macaque IT cortex is of high importance to interpret the results obtained with the fMRI adaptation paradigm in normal and patient populations (Ewbank et al., 2015) and is essential when examining the ventral visual pathway. Furthermore, adaptation is an example of the impact of stimulus history on neural stimulus representations. Given that stimulus history affects percep- 
tion (Bar and Biederman, 1999; Müller et al., 2009; Noudoost and Esteky, 2013; Dekel and Sagi, 2015), a better understanding of adaptation is necessary to gain a deeper insight into the neural mechanisms of perception (Clifford et al., 2007).

The adaptation effects observed at the level of spiking activity of single neurons can be viewed as resulting from adaptation effects that occur in the network of neurons in which the neuron is embedded. This network view of adaptation can explain why adaptation in the primary visual cortex (V1) results in less suppression or in some cases even leads to enhancement for large compared with small stimuli (Wissig and Kohn, 2012; Patterson et al., 2013). Such response enhancement has been hypothesized to result from adaptation of the inhibitory extraclassical receptive field input ("surround") to the neuron. These and other data obtained in the V1 can be explained by assuming adapted inputs in a divisive normalization circuit (Dhruv et al., 2011; Solomon and Kohn, 2014). Divisive normalization is a computation implemented by neural circuits in which the response of a neuron is determined by a driving, afferent input and a divisive input from other neurons within the network that respond to the stimulus. Divisive normalization has been successfully applied to model a variety of neuronal response properties, including contrast response nonlinearity, nonlinear spatial summation, and cross-orientation inhibition at early stages of the visual system (Carandini and Heeger, 2012), as well as spatial attentional effects (Reynolds and Heeger, 2009; Ni et al., 2012) and multisensory integration (Ohshiro et al., 2011).

The responses of single IT cortex neurons to a complex stimulus configuration have been demonstrated to be less than the sum of the responses to its constituent parts (Richmond et al., 1983; Sato, 1989; Miller et al., 1993; Rolls and Tovee, 1995; Missal et al., 1999) and in fact has been reported to be in good correspondence with the average of the responses to the constituent parts presented in isolation (Zoccolan et al., 2005; De Baene et al., 2007). This interaction between the different parts of a complex stimulus configuration can be understood as resulting from a normalization of the bottom-up input to a neuron by a divisive input from a population of other IT cortex neurons that respond to such a complex stimulus. Both the bottom-up and the divisive normalizing inputs are in principle susceptible to adaptation. The interplay between these two inputs in combination with differential adaptation of each of them can in theory produce a variety of adaption effects, ranging from suppression to enhancement of the response following adaptation. In the present study, we examined how adaptation of each putative type of input (i.e., driving bottom-up vs divisive normalizing) to a neuron affects stimulus interactions of single macaque IT cortex neurons for compound stimuli consisting of the simultaneous presentation of two stimuli. To address this question, we derived predictions from a divisive normalization model and then tested those using recordings of single-unit spiking activity in the macaque IT cortex.

\section{Materials and Methods}

\section{Subjects}

Two rhesus macaques (Macaca mulatta; male Monkey P and female Monkey K, weighing 7.2 and $7.6 \mathrm{~kg}$, respectively, both left hemisphere) served as subjects. Animal care and experimental procedures met the national and European guidelines and were approved by the Ethical Committee of the KU Leuven Medical School.

Details about implants and surgery can be found in Kaliukhovich and Vogels (2011) and will only be briefly summarized here. The placement of the plastic recording chamber was guided with a preoperative MRI scan and verified with MRI scans obtained at the beginning and in between recording sessions. Reliable estimations of the recording positions were obtained by the visualization of glass capillaries, filled with the MRI opaque copper sulfate $\left(\mathrm{CuSO}_{4}\right)$, that were inserted into the recording chamber grid (Crist Instrument) at predetermined positions. Recording positions were estimated based on the MRI visualization of these markers combined with the microdrive depth readings of the white/gray matter transitions relative to the grid base.

Recordings were made from the lower bank of the superior temporal sulcus and lateral convexity of the IT cortex. The anterior-posterior coordinates of the estimated recording positions ranged between 18 and $20 \mathrm{~mm}$, and 17 and $18 \mathrm{~mm}$ anterior to the auditory meatus in Monkeys $\mathrm{P}$ and $\mathrm{K}$, respectively, whereas the corresponding medial-lateral coordinates in those monkeys ranged between 18 and $20 \mathrm{~mm}$ and 20 and $24 \mathrm{~mm}$ lateral to the midline.

\section{Recordings}

Well-isolated extracellular action potentials of single IT cortex neurons were recorded with tungsten microelectrodes. The electrode was lowered with a Narishige microdrive through a guide tube that was fixed in a Crist grid. Recordings were made using a Plexon data acquisition system. Spiking activity was thresholded on-line and spike waveforms were saved at $40 \mathrm{kHz}$. Spikes were sorted when necessary and single-unit isolation was verified using Offline Sorter (Plexon).

Eye position was measured on-line with an infrared-based eyetracking system (EC-240A, ISCAN; $120 \mathrm{~Hz}$ sampling rate). The analog eye-movement signal was saved with a sampling frequency of $1 \mathrm{kHz}$. Eye positions, stimulus events, and behavioral events were recorded simultaneously with spiking activity and stored for later off-line analysis on a computer synchronized with the Plexon data acquisition system.

\section{Stimuli and tests}

Stimuli. In the present study we used a stimulus set identical to that used in our previous studies (Kaliukhovich and Vogels, 2012, 2014; Kaliukhovich et al., 2013) on adaptation in the macaque IT cortex. The stimulus set consisted of 52 color images of 26 object classes (two images per class), including human and monkey faces, human and monkey bodies, mammals, birds, fish, snakes, insects, trees, fruits, fractals, and manmade objects. The size of the stimuli (maximum of horizontal and vertical dimensions of the bounding box) was $\sim 5^{\circ}$ of visual angle. The stimuli were presented on a uniform gray background on a CRT monitor (Philips Brilliance 202P4; frame rate, $60 \mathrm{~Hz}$; resolution, $1024 \times 768$ pixels) located $60 \mathrm{~cm}$ from the subject's eyes.

Search test. We used this test to search for responsive neurons while advancing a microelectrode in the IT cortex. Once the spiking activity of a responsive neuron was well isolated, the search test was further used to select two stimuli for the neuron, with one of the two stimuli evoking a strong response (effective stimulus) and the other little or no response at all (ineffective stimulus). The two stimuli were selected from the pool of 52 color images (see Stimuli).

On each trial of the search test, the monkeys were required to passively maintain their gaze on a red fixation target square (size, $0.17^{\circ}$ ) presented in the center of the monitor and visible during the entire trial. A trial started with the onset of the fixation target. After $500 \mathrm{~ms}$ of stable fixation, a stimulus was presented for $500 \mathrm{~ms}$. To complete a trial and obtain a fluid reward, the monkeys had to maintain fixation for $475 \mathrm{~ms}$ poststimulus. The different images (see Stimuli) were presented foveally in a random manner. Selection of the effective and ineffective stimuli for an examined neuron was based on the peristimulus time histograms available on-line and computed with $\geq 1$ presentation (mean, 5.5) per tested stimulus.

Adaptation test. After we selected the effective and ineffective stimuli for a neuron under study in the search test, we ran the adaptation test (Fig. 1A). On each trial of the adaptation test, monkeys were required to passively fixate a red target square $\left(\right.$ size, $\left.0.17^{\circ}\right)$ shown in the center of the monitor and visible during the entire trial. Following $500 \mathrm{~ms}$ stable fixation, monkeys were presented with two stimuli, with each stimulus being presented for $300 \mathrm{~ms}$. The two stimuli were separated by a blank screen (and fixation target) for $500 \mathrm{~ms}$. To complete a trial and obtain a 
fluid reward, monkeys had to maintain their fixation for another $300 \mathrm{~ms}$ after offset of the test stimulus. Any break in fixation during this $1900 \mathrm{~ms}$ interval aborted the trial. The extent of the eye-position signal in unaborted trials, computed per neuron, ranged from 0.6 to $1.2^{\circ}$ horizontally and from 0.8 to $1.7^{\circ}$ vertically across all the analyzed neurons of both monkeys. This indicates more accurate fixation than the $2^{\circ}$-square fixation window. Each trial ended with the presentation of a full-field scrambled stimulus for $500 \mathrm{~ms}$ followed by a blank screen for $1000 \mathrm{~ms}$. The scrambled stimulus was randomly chosen from a pool of 100 such stimuli generated by scrambling 20 color scenes (size, $1024 \times 768$ pixels) with $32 \times 32$ pixel-wide square elements. Each scene was scrambled five times $(20$ scenes $\times 5=100$ stimuli).

We tested the following adaptation conditions (Fig. 1B): (1) repetition of the effective stimulus (Fig. 1B, E-E); (2) repetition of the ineffective stimulus (I-I); (3) repetition of the compound stimulus (C-C), which was a simultaneous presentation of both the effective and ineffective stimuli; (4) effective stimulus followed by the compound stimulus (E-C); and (5) ineffective stimulus followed by the compound stimulus (I-C). Single (effective or ineffective) stimuli were presented either above or below the fixation target along the vertical meridian, with their center of mass being positioned at an eccentricity of $3^{\circ}$. For the compound stimuli, one stimulus was presented above and the other below the fixation target. Once presented, the position of a stimulus remained the same in a trial. Given that either single stimulus could have been presented at one of the two positions (above or below the fixation target), this resulted in total in 10 stimulus conditions ( 5 conditions $\times 2$ positions). During the recordings, these 10 stimulus conditions were presented to the monkeys in a randomly interleaved manner. The minimum and mean number of presentations per stimulus condition across all analyzed neurons of both monkeys was 9 and 18.4, respectively.

\section{Data analysis}

For each recorded neuron and stimulus condition (Fig. 1), we computed the mean firing rate to the adapter and test stimuli. Only unaborted trials were analyzed. Responses to the stimuli were computed within a $300 \mathrm{~ms}$ analysis window starting at $50 \mathrm{~ms}$ after stimulus onset. Only neurons that showed a significant excitatory (more than baseline) response to the effective stimulus at either position were analyzed further. Significance at each position was tested by comparing the response to the effective stimulus presented as adapter in repetition trials with the baseline activity within the $100 \mathrm{~ms}$ window directly preceding the adapter stimulus onset (Bonferroni-corrected one-sided Wilcoxon matched-pairs test, $p<$ $0.05 / 2$ positions $=0.025$ )

Except for the compound stimuli, both effective and ineffective stimuli were presented at each of the two positions (above or below the fixation target). Most but not all neurons responded to the effective stimulus at both positions (see Results). Here we analyzed and presented the data using two approaches. The first approach was based on the analysis of responsive position $\times$ neuron combinations. Specifically, for an examined neuron, the data for each of the two positions were analyzed separately, and only those positions per neuron were taken that showed a significant excitatory response to the effective stimulus. Thus, depending on the response to the effective stimulus at each position, each neuron could contribute to the analyses one or maximum two responsive posi-

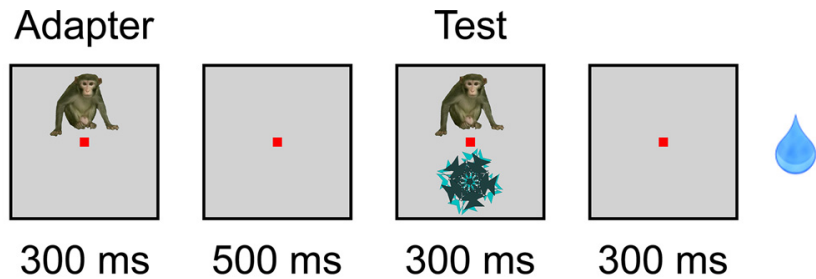

Position 1
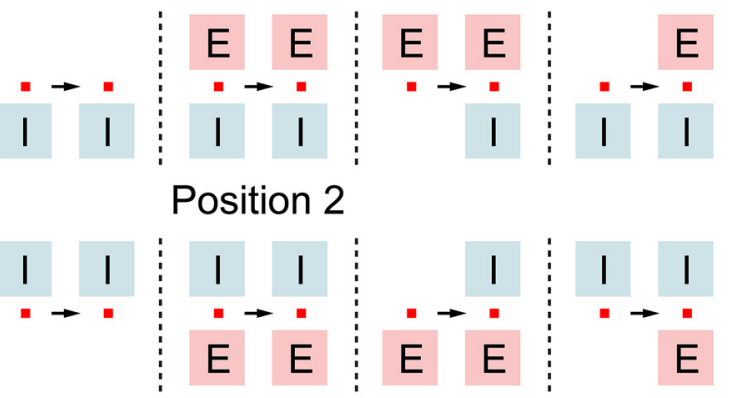

I-I

C-C

E-C

I-C

Figure 1. Experimental paradigm and stimulus conditions. $A, 0$ n each trial, monkeys were required to fixate a red target square (here shown not to scale; size: $0.17^{\circ}$ of visual angle) shown in the center of the monitor during the entire trial. Monkeys initiated al by fixating the target. After fixation of the target for $500 \mathrm{~ms}$, the monkeys were presented with two stimuli, with the firs of a trial is further referred to as the adapter stimulus, with the second stimulus referred to as the test stimulus. To complete the trial extent: $5^{\circ}$ of visual angle) were selected in such a manner that when presented in isolation, one image strongly drove the neuron (effective (E)], whereas the other evoked little or no response [ineffective (I)]. The images were presented along the eccentricity of $3^{\circ}$ of visual angle. The simultaneous presentation of both effective and ineffective images is further referred to as the (the position of either image (E or I) remained the same in a trial. Stimulus conditions included repetition of the effective image (E-E), of the ineffective image (I-I), or of the compound stimulus $(C-C)$, as well as presentation of the compound stimulus following either the effective $(\mathrm{E}-\mathrm{C})$ or ineffective $(\mathrm{I}-\mathrm{C})$ image. Given that both images (E or I) could be presented at either position, this resulted in a total of 10 stimulus conditions.

tion $\times$ neuron combinations. In the second approach, for each neuron we averaged the data across responsive positions per stimulus condition. Here we used the first approach for descriptive statistics given its problem of artificially inflated degrees of freedom. Indeed, the effect sizes of two responsive position $\times$ neuron combinations derived from the same neuron may correlate, thus potentially violating the assumption of independent observations inherent to the standard statistical tests. As a result, the degrees of freedom, as judged from the number of analyzed position $\times$ neuron combinations, can be overestimated. Thus, for statistical tests we used the second approach, in which the number of observations equals the number of neurons.

In all reported analyses, unless otherwise stated, we used the mean gross responses. The same analyses performed on baseline-corrected, net responses showed qualitatively the same effects as those based on gross responses.

\section{Modeling}

We fitted the responses of a neuron with divisive normalization models. In the models, a neuron receives a direct, bottom-up input from neurons that respond to features that are part of the effective or ineffective stimulus. To model the suppressed activity for the compound relative to the effective stimulus, the neuron also receives a divisive, normalizing input from a population of IT cortex neurons that responds to both the effective and ineffective stimuli. In the first model, the "adapted driving and normalizing input" (ADNI) model, each of the bottom-up inputs (Eff and InEff for the effective and ineffective stimuli, respectively) can become suppressed following adaptation. We modeled such suppression by equally scaling these inputs by a factor $\beta$. Thus, adaptation to the effective stimulus will result in a $1 / \beta$-fold decrease of Eff without affecting InEff. 
Table 1. Modeled stimulus conditions and the corresponding values of the dummy variables $^{a}$

\begin{tabular}{llllll}
\hline Modeled stimulus condition & Condition label & $U$ & $V$ & $W$ & Z \\
\hline $\begin{array}{l}\text { Effective stimulus presented as } \\
\text { adapter }\end{array}$ & Adapter (E-E) & 1 & 0 & 0 & 0 \\
$\begin{array}{l}\text { Ineffective stimulus presented } \\
\text { as adapter }\end{array}$ & Adapter (I-I) & 0 & 0 & 1 & 0 \\
$\begin{array}{c}\text { Compound stimulus presented } \\
\text { as adapter }\end{array}$ & Adapter (C-C) & 1 & 0 & 1 & 0 \\
$\begin{array}{l}\text { Repeated effective stimulus } \\
\text { Repeated ineffective stimulus }\end{array}$ & Test (E-E) & 1 & 1 & 0 & 0 \\
$\begin{array}{l}\text { Repeated compound stimulus } \\
\text { Compound stimulus following } \\
\text { the effective stimulus }\end{array}$ & Test (C-C) & 0 & 0 & 1 & 1 \\
$\begin{array}{c}\text { Compound stimulus following } \\
\text { the ineffective stimulus }\end{array}$ & Test (I-C) & 1 & 1 & 1 & 1 \\
\hline
\end{tabular}

${ }^{a}$ The same dummy variables and their values were used to model neuronal responses in both divisive normalization models. The labels "Adapter" and "Test" in the column "Condition label" refer to the adapter and test stimuli in a trial (Fig. 1A), whereas $\mathrm{E}, \mathrm{l}$, and $\mathrm{C}$ in the same column correspond to the effective, ineffective, and compound stimuli, respectively. See a full list of stimulus conditions in Figure $1 B$.

Conversely, adaptation to the ineffective stimulus will scale down InEff by a factor of $1 / \beta$ without affecting Eff. Similar to the direct input, the divisive normalizing input is also susceptible to adaptation in the ADNI model. Thus, adaptation to the effective or ineffective stimuli will reduce that divisive input by a factor $\gamma$, with $\gamma=1$ corresponding to no adaptation of the divisive input and $\gamma<1$ indicating a suppressed divisive input. The adaptation parameters $\beta$ and $\gamma$ were constrained to vary between 0 and 1 . We implemented tuned normalization (Ni et al., 2012) by using a parameter $\alpha$, allowing variation in the contribution to the normalization by the ineffective stimulus relative to the effective stimulus. The parameter $\alpha$ could vary from 0 (no effect of the ineffective stimulus to response normalization) to infinity (negligible effect of the effective stimulus to response normalization). Note that an $\alpha$ value of 1 implies equal influence of both stimuli. Two additional parameters were the semisaturation constant $\sigma$, set to 0.5 , and the baseline activity $\delta$, which was fixed for each modeled neuron and computed as the average baseline activity across all stimulus presentations (regardless of stimulus condition) of that neuron. Importantly, the results of modeling did not depend qualitatively on a particular choice of the semisaturation constant $\sigma$ and also remained qualitatively similar when using other plausible ranges of $\alpha$, such as from 0 to 5 , from 0 to 10 , and from 0 to 100 .

Thus, the response of a neuron in the ADNI model was computed as follows (Eq. 1): $\operatorname{resp}_{\mathrm{ADNI}}=\left(U \cdot \beta^{V} \cdot\right.$ Eff $+W \cdot \beta^{Z} \cdot$ Ineff $) /\left(U \cdot \gamma^{V}+W \cdot\right.$ $\left.\alpha \cdot \gamma^{Z}+\sigma\right)+\delta$, where $U, V, W$, and $Z$ are dummy variables that took on the values of 0 or 1 , depending on the particular stimulus condition being modeled (Table 1). For instance, $V$ and $Z$ took on the value 1 if a test stimulus followed the effective or ineffective stimulus, respectively, and 0 otherwise. Similarly, $U$ and $W$ took on the value 1 if the modeled stimulus condition included a presentation of the effective or ineffective stimulus, respectively, and 0 otherwise. The input parameter Eff was constrained to lie between 0 and 10 times the measured gross response to the single effective stimulus presented as an adapter to the neuron at the modeled location in repetition trials. The input parameter InEff could vary between -10 times and +10 times the gross response to the effective stimulus. Thus, as observed in some neurons (see Results), presentation of the ineffective stimulus could inhibit the response.

The second model, the "adapted driving input" (ADI) model, allowed adaptation to occur for the driving inputs Eff and InEff, i.e., for the nominator of the divisive normalization model, but not for the divisive normalizing input, i.e., the denominator. In addition, the degree of adaptation was allowed to differ between the excitatory Eff input and the potentially inhibitory InEff input. Such differential adaptation of each driving input was achieved by using two independent adaptation parameters, $\beta$ and $\mu$. The response of a neuron in the ADI model was computed as follows (Eq. 2): $\operatorname{resp}_{\mathrm{ADI}}=\left(U \cdot \beta^{V} \cdot\right.$ Eff $+W \cdot \mu^{Z} \cdot$ Ineff $) /(U \cdot 1+W \cdot$ $\alpha+\sigma)+\delta$, with all parameter settings, constraints, and dummy variables being identical to those of the first ADNI model. Note that both models included the same number of free parameters (Eff, InEff, $\beta, \gamma, \alpha$ vs Eff, InEff, $\beta, \mu, \alpha$ ), thus, allowing an easy comparison in goodness of fit between the two models.

We modeled the raw response to the following eight stimulus conditions (Table 1 ) for each position $\times$ neuron combination: the effective stimulus presented as adapter in repetition trials, the ineffective stimulus presented as adapter in repetition trials, the compound stimulus presented as adapter in repetition trials, the repeated effective stimulus, the repeated ineffective stimulus, the repeated compound stimulus and, last, the compound stimulus following either the ineffective or effective stimulus. To fit the two models to the responses in the stimulus conditions for each analyzed position $\times$ neuron combination, we used the Matlab function "lsqcurvefit," which performs least-squares nonlinear data fitting. To minimize the sum of squared residuals between the observed and predicted responses, we used the trust-region reflective algorithm with the following parameters: termination tolerance on the estimated parameters $(\mathrm{TolX})=10^{-6}$, termination tolerance on the minimized function (the sum of squared residuals, TolFun) $=10^{-6}$, allowed maximum number of iterations (Maxiter) $=10,000$, allowed maximum number of evaluations of the minimized function (MaxFunEvals) $=50,000$. The fitting of the model parameters was done 10,000 times for each position $\times$ neuron combination. Importantly, each time we used a new set of randomly drawn initial values of the free parameters (within their predefined constraints), and these were identical for the two models, with initial values of the adaptation parameter $\gamma$ of the ADNI model being matched to the adaptation parameter $\mu$ of the ADI model. We took the fit with the minimum sum of squared errors (which is identical to the highest coefficient of determination) over the set of 10,000 solutions as the final fit. In practice, the range of the fitted parameters for a particular position $\times$ neuron combination was, however, quite small and negligible. We used $r^{2}$, defined as $1-$ (residual sum of squares/total sum of squares) as measure of the goodness of fit.

\section{Analysis of eye movements}

For each analyzed neuron and stimulus condition, we computed the mean eye-position signal along the horizontal and vertical axes, and this separately for the adapter and test stimuli. As in the analyses of spiking activity, only unaborted trials were selected. For each analyzed neuron, we next computed the mean baseline eye position by averaging the eyeposition signal within a $300 \mathrm{~ms}$ window before adapter onset across the 10 stimulus conditions (Fig. 1B). This was done separately for the horizontal and vertical axes. Then, for each analyzed neuron, we further subtracted the thus derived mean baseline eye position from each stimulus condition, and this separately for each axis. This baseline correction-based on the eye position before adapter onsetexplains why the eye positions for the compound test stimulus shown in Figure 7 can be $<0$.

To examine whether the position of the adapter stimulus systematically affected eye movements and thus may explain the effects observed at the level of spiking activity, we compared eye-position signals for the compound stimulus presented as test when following the adapter stimulus presentation in the upper versus lower visual field. Given that each of the two positions encompassed presentations of both the effective and ineffective stimuli for each analyzed neuron and position (above and below the fixation target), we averaged the baseline-corrected mean eyeposition signal for the compound stimulus presented as test across the two stimuli (stimulus conditions E-C and I-C; Fig. 1B). Similarly, by averaging across the two positions we computed the mean eyemovement signal for the compound stimulus presented as test when following the same compound stimulus (stimulus condition C-C; Fig. $1 B$ ). To examine the effect of the adapter stimulus position on eye movements at the population level in each monkey separately, we used the same selections of neurons as in the analyses of spiking activity. Here we only report the results based on the analysis of eye movements along the vertical axis, because no effects were found along the horizontal axis.

\section{Results}

We recorded the responses of 81 single macaque IT cortex neurons to stimuli presented at two eccentric positions along the 


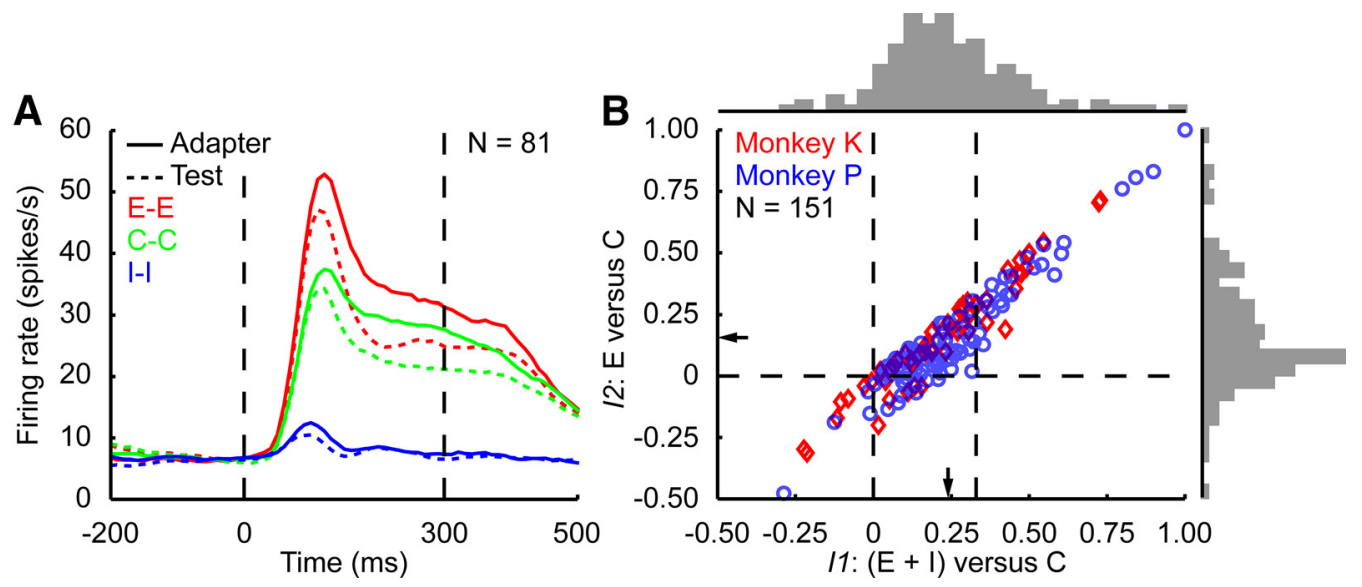

Figure 2. Responses to the effective, ineffective, and compound stimuli compared. $A$, Population peristimulus time histograms of the responses to the effective (red), ineffective (blue), and compound (green) stimuli presented as adapter (solid line) and test (stippled line) in repetition trials. The responses were first averaged across responsive positions for each neuron and then averaged across neurons of both monkeys. Peristimulus time histograms for each stimulus condition and neuron were constructed by applying a running average with a width of the boxcar kernel of $50 \mathrm{~ms}$ and a step of $10 \mathrm{~ms}$. Mean spiking activity is plotted at the center of the corresponding time bins. Stippled vertical lines indicate stimulus onset and offset. $N$ denotes the number of neurons. $B$, The response to the compound and single stimulus presentations compared. Indices $/ 1$ and $/ 2$ were computed for each responsive position $\times$ neuron combination (see Materials and Methods) and then pooled across both monkeys (Monkey K: $N=56$, red; Monkey $P: N=95$, blue). 11 compares the response to the compound stimulus with the sum of the responses to the two constituent stimuli when the latter are presented in isolation. 12 compares the response to the compound stimulus with the response to the effective stimulus when presented alone. The marginal distributions are shown in gray, and the mean values are indicated by arrows. The stippled vertical line at $/ 1=0.33$ corresponds to the expected value when the response to the compound stimulus is the average of the responses to the effective and ineffective stimuli. $N$ denotes the total number of position $\times$ neuron combinations across both monkeys.

vertical meridian of the visual field. The stimuli were selected in a search test (see Materials and Methods). In this test, we foveally presented 52 color images of objects of different categories to the monkeys. For each neuron under study, we selected two images, with one image evoking a strong response (effective stimulus) and the other no or little response (ineffective stimulus). The two images differed across neurons due to the different stimulus selectivity of the neurons. Once we identified the effective and ineffective stimuli for a recorded neuron, we next performed the adaptation test used to address the research question of the present study. In the adaptation test, the two selected stimuli were shown for $300 \mathrm{~ms}$, either separately (single stimulus) or in combination (compound stimulus), in a sequence of two presentations separated by an interstimulus interval of $500 \mathrm{~ms}$ (Fig. 1; see Materials and Methods). The first presentation in the sequence will be labeled as "adapter" and the second as "test." Statistical testing (see Materials and Methods) showed that 81 IT cortex neurons recorded in two rhesus monkeys (Monkey P: $N=51$; Monkey K: $N=30$ ) responded significantly to the effective stimulus when presented alone at at least one of the two eccentric positions. Of these 81 neurons, the large majority (70) showed a significant response to the effective stimulus at both eccentric positions. When considering each position separately (two positions per neuron), this yielded 151 cases (position $\times$ neuron combinations; Monkey P: $N=95$; Monkey K: $N=56$ ) for which there was a significant excitatory response to the effective stimulus at a tested position. These were divided nearly equally across the two positions (upper visual field: $N=75$; lower visual field: $N=76)$.

\section{Responses to compound and single stimuli compared}

The mean gross response to the effective stimulus presented as adapter in repetition trials was 35.3 spikes/s, whereas the mean gross response to the ineffective stimulus was 8.9 spikes/s (net

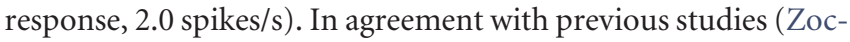
colan et al., 2005; De Baene et al., 2007), the response to the compound stimulus (mean gross response when presented as adapter in repetition trials, 28.5 spikes/s) was significantly lower than the response to the effective stimulus presented alone (Fig. 2A; two-sided Wilcoxon matched-pairs test: $p<0.000001$, $N=81$ neurons), and this held true for each monkey. This was not merely due to suppression by inhibitory ineffective stimuli because restricting this comparison to the cases $(N=88$ position $\times$ neuron combinations) for which the net response to the ineffective stimulus was $\geq 0$ spikes/s produced similar results (mean gross response: effective stimulus, 40.7 spikes/s; compound stimulus, 34.1 spikes/s).

To quantify the interaction between the effective and ineffective stimuli, we next computed two indices for each of the 151 responsive position $\times$ neuron combinations as follows: $I 1=[\mathrm{re}$ $s p(E)+\operatorname{resp}(I)-\operatorname{resp}(C)] /[\operatorname{resp}(E)+\operatorname{resp}(I)+\operatorname{resp}(C)]$ and $I 2=[\operatorname{resp}(E)-\operatorname{resp}(C)] /[\operatorname{resp}(E)+\operatorname{resp}(C)]$, where $\operatorname{resp}(X)$ denotes the response to stimulus $X$, and $E, I$, and $C$ correspond to the effective, ineffective, and compound stimuli, respectively. The responses were computed within a $300 \mathrm{~ms}$ analysis window (see Materials and Methods). I1 compares the response to the compound stimulus with the sum of the responses to the two constituent (effective and ineffective) stimuli when the latter are presented in isolation. I2 compares the response to the compound stimulus with the response to the effective stimulus when presented alone, thus capturing suppression of the response to the effective stimulus due to a simultaneous presentation of the ineffective stimulus. As is expected from the weak responses to the ineffective stimulus, $I 1$ and $I 2$ were highly correlated across neurons (Fig. 2B; Pearson correlation: $r=0.95$ ). The distributions of the two indices (Fig. $2 B$ ) showed that the responses of most neurons to the compound stimulus were suppressed (mean $I 2=0.16, N=151$ position $\times$ neuron combinations) when compared with the responses to the effective stimulus. The mean I1 was 0.24 , which was lower than the expected value of 0.33 if averaging of the responses to constituent parts was the mechanism determining the response to the compound stimulus (Zoccolan et al., 2005; De Baene et al., 2007). Interestingly, a few position $\times$ neuron combinations showed similar responses to the 


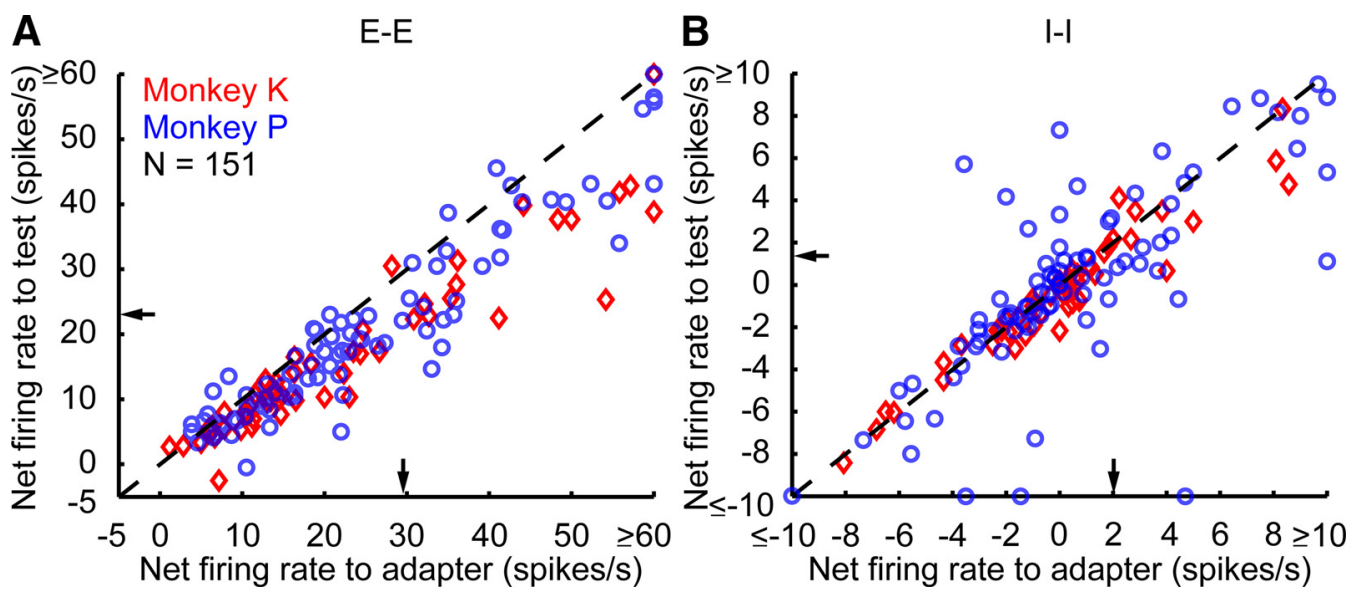

Figure 3. Repetition suppression for single effective and ineffective stimuli. $\boldsymbol{A}, \boldsymbol{B}$, The scatter plots show the net firing rate for a stimulus ( $\boldsymbol{A}$, effective; $\boldsymbol{B}$, ineffective) in repetition trials presented as adapter versus test (Fig. 1B). Each point corresponds to a single position $\times$ neuron combination. Data of the two animals were pooled and indicated by different colors and symbols. $N$ denotes the total number of position $\times$ neuron combinations, whereas arrows indicate the mean values. Note the smaller range of the axes in $\boldsymbol{B}$ compared with $\boldsymbol{A}$.

effective and compound stimuli $(I 2=0)$, which corresponds to a winner-take-all mechanism.

\section{Repetition suppression}

In agreement with previous work (Miller et al., 1991; Vogels et al., 1995; Sawamura et al., 2006; McMahon and Olson, 2007; Liu et al., 2009; De Baene and Vogels, 2010; Kaliukhovich and Vogels, 2011, 2012, 2014), repetition of the single effective stimulus suppressed the response (Fig. $3 A$ ). The mean gross response to the effective stimulus (300 ms analysis window; see Materials and Methods) presented as adapter in repetition trials was 35.3 spikes/s, which was significantly greater than the mean gross response of 28.8 spikes/s to the repeated effective stimulus (responses were first averaged across responsive positions per neuron; two-sided Wilcoxon matched-pairs test: $p<0.000001$, $N=81$ neurons). This repetition suppression was statistically significant in each monkey (both $p$ values $<0.00001$ ).

Repetition of the single ineffective stimulus also resulted in response suppression (mean gross response in repetition trials when using a $300 \mathrm{~ms}$ analysis window: adapter, 8.7 spikes/s; test, 7.9 spikes/s; two-sided Wilcoxon matched-pairs test: $p<0.05$, $N=81$ neurons). Similar results were observed when analyzing net (baseline-corrected) responses per position $\times$ neuron combination for the single effective (Fig. $3 A$ ) and ineffective (Fig. 3B) stimuli in repetition trials. Specifically, the mean response to either the single effective or single ineffective stimulus presented as adapter was significantly greater than the response to the same stimulus presented as test.

\section{Predicted responses to the compound stimulus following adaptation: simulations}

We next examined how adaptation to the single (effective and ineffective) and compound stimuli affected the response to the compound stimulus. To predict such adaptation-induced effects, we first performed simulations with the divisive normalization ADNI model that implements tuned normalization (Eq. 1). In this model, a neuron receives a direct, bottom-up excitatory input from the neurons that respond to features that are part of the effective or ineffective stimulus. Each of these bottom-up inputs (one for the effective and another for the ineffective stimulus) can become equally suppressed following adaptation. We modeled such suppression by scaling these inputs by a factor $\beta$ (Eq. 1). In the model, the neuron also receives a divisive, normalizing input from a population of IT cortex neurons that responds to both the effective and ineffective stimuli. Similar to the direct input, we assume that the divisive normalizing input is also susceptible to adaptation. Adaptation to the effective or ineffective stimuli will reduce that divisive input by a factor of $\gamma$ (Eq. 1).

Figure $4 A-C$ shows the outcome of the ADNI model applied for three adaptation conditions, when varying $\beta$ and $\gamma$ between 0.01 (strong suppression) and 1 (no suppression). In the reported simulations, the tuned normalization parameter $\alpha$ (see Materials and Methods) was set to 0.75 , but the trends remained the same for other values of $\alpha$ (including an equal degree of normalization of the two inputs). The input parameters Eff and InEff were set to 30 and 1 , respectively, whereas the baseline $\delta$ was 0 . The outcomes of the simulations are presented as contrast indices (CIs) computed as follows: $C I=[\operatorname{resp}(i)-\operatorname{resp}(j)] /[\operatorname{resp}(i)+\operatorname{resp}(j)]$, with $\operatorname{resp}(X)$ being the simulated response to stimulus $X$, and $i$ and $j$ being the stimuli in the conditions defined in Figure 4 (see $i$ vs $j$ ). The comparison of the simulated responses to the compound stimulus presented as adapter and test in repetition trials (Fig. $4 A$ ) reveals response suppression $(C I>0)$ for the repeated compound stimulus, except when the divisive input is suppressed more than the direct one $(\gamma<\beta)$. Figure $4 B$ compares the simulated response to the repeated compound stimulus with the response to the same compound stimulus following adaptation to an effective stimulus. Overall, this comparison shows a smaller response to the compound stimulus adapted to the effective stimulus than to the same repeated compound stimulus $(C I>0)$, except when adaptation of the direct input is much stronger than that of the divisive input $(\gamma \gg \beta)$. Last, adaptation to an ineffective stimulus enhances the simulated response to the compound stimulus compared with when the latter is presented as adapter $(C I>0$; Fig. $4 C)$. This enhancement is present for all combinations of $\beta$ and $\gamma$, except for the values of $\gamma$ close to 1 (no suppression of the divisive input). Based on these simulations of the divisive normalization model that includes adaptation of the direct and divisive inputs, we predict (1) repetition suppression for compound stimuli, (2) stronger repetition suppression when adapting to the effective compared with the compound stimulus and, importantly, (3) an enhancement of the response to the compound stimulus following adaptation to an ineffective stimulus. These predictions hold over a wide region of the $\beta-\gamma$ space (region within stippled black lines in Fig. 4A-C), including those in which adaptation of the direct and divisive inputs is equally 

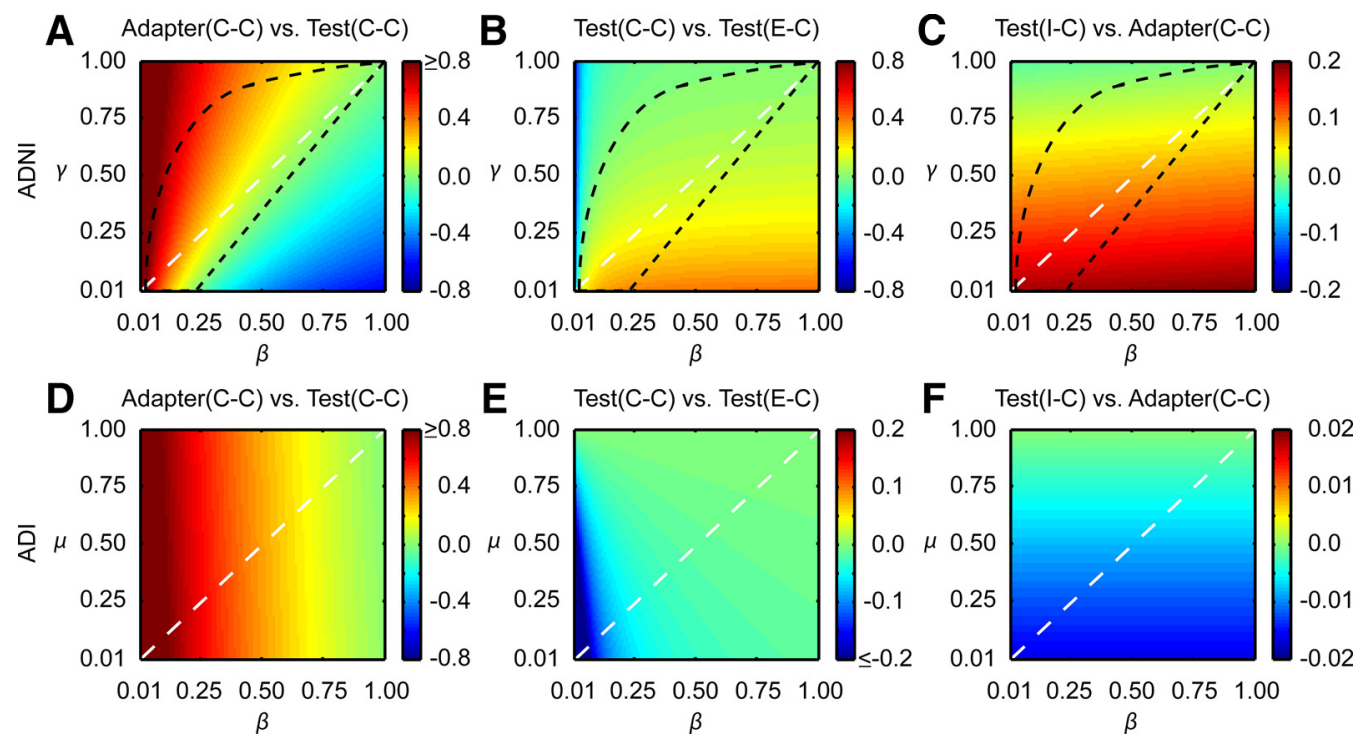

Figure 4. $\quad \boldsymbol{A}-\boldsymbol{F}$, Prediction of adaptation-induced effects for both ADNI $(\boldsymbol{A}-\boldsymbol{C})$ and ADI $(\boldsymbol{D}-\boldsymbol{F})$ models. For each stimulus condition and model, we computed the responses to the adapter and test stimuli (Eqs. 1 and 2). For each model separately, we then compared the responses to the compound stimulus presented as adapter and test in repetition trials $(\boldsymbol{A}, \boldsymbol{D})$, when being repeated versus following adaptation to the effective stimulus $(\boldsymbol{B}, \boldsymbol{E})$, and when following adaptation to the ineffective stimulus versus being presented as adapter $(\boldsymbol{C}, \boldsymbol{F})$. To compare the responses, we computed contrast indices while varying the gain of direct $(\beta)$ and divisive $(\gamma)$ inputs in the ADNI model and of only the direct input for each stimulus independently $(\beta$ and $\mu$ for the effective and ineffective stimuli, respectively) in the ADI model. A positive value for a pair $X v$ v $Y$ corresponds to a higher response to the compound stimulus in condition $X$ than in $Y$, with the opposite being true for negative values. The region indicated by the stippled black lines in $\boldsymbol{A}-\boldsymbol{C}$ corresponds to the intersection of the regions in those panels with positive contrast indices in the ADNI model. Given that the contrast indices in $\boldsymbol{E}$ and $\boldsymbol{F}$ were all $\leq 0$, we failed to derive a similar region for the $\mathrm{ADI}$ model.

strong $(\beta=\gamma)$. Note that the exact boundaries of the $\beta-\gamma$ region depend on the values of the input parameters.

We further examined the predictions for adaptation-induced effects derived from the ADI model. Similar to the ADNI model, in the ADI model a neuron also receives direct, bottom-up inputs due to presentation of the effective (Eff) and ineffective (InEff) stimuli, with each type of input (Eff or InEff) being susceptible to adaptation. Yet, in contrast to the ADNI model, which implements the same degree of adaptation $(\beta$; Eq. 1$)$ on each input (both Eff and InEff), the ADI model allows each input to be adapted separately of the other by using two independent adaptation parameters ( $\beta$ and $\mu$; Eq. 2 ). Last, both the ADNI and ADI models incorporate a tuned divisive, normalizing input from a population of IT cortex neurons that responds to both the effective and ineffective stimuli, with only the ADNI model and not the ADI model allowing this normalizing input being also susceptible to adaptation.

Figure $4 D-F$ shows the outcome of the ADI model applied for the same three adaptation conditions as those examined in the ADNI model. When computing the responses in the ADI model (Eq. 2; Table 1), we used the same values of the parameters as those used to compute the responses in the ADNI model $(E f f=30 ; \operatorname{InEff}=1 ; \alpha=0.75 ; \sigma=0.5, \delta=0)$, while allowing the adaptation parameters $\beta$ and $\mu$ to vary between 0.01 (strong suppression) and 1 (no suppression). The ADI model predicted a smaller or equal response to the repeated compound stimulus than to the same compound stimulus presented as adapter for the entire examined $\beta-\mu$ space (all $C I s \geq 0$; Fig. $4 D$ ). Moreover, the ADI model predicted a smaller or equal response to the repeated compound stimulus than to the same compound stimulus following adaptation to the effective stimulus for the entire $\beta-\mu$ space (all CIs $\leq 0$; Fig. $4 E$ ). Note that this is the opposite of the prediction derived from the ADNI model: namely, a stronger response suppression when adapting to the effective compared with the compound stimulus (Fig. 4, compare $B, E$ ). Last, adap- tation to the ineffective stimulus in the ADI model resulted in a smaller or equal response to the compound stimulus compared with the response to the same compound stimulus presented as adapter $(C I \leq 0$ for the entire $\beta-\mu$ space; Fig. $4 F)$, which is the opposite of the prediction of the ADNI model (Fig. 4, compare $C$, $F)$. In conclusion, apart from response suppression to the repeated compound stimulus, these simulations of the ADNI and ADI models make different predictions for adaptation-induced effects for the two pairs of the compared stimulus conditions [Test(C-C) vs Test(E-C) and Test(I-C) vs Adapter(C-C)].

\section{Observed responses to the compound stimulus following adaptation}

Figure $5 \mathrm{~A}$ shows the mean gross responses to the compound stimulus following adaptation to either a single (effective or ineffective) stimulus or the same compound stimulus. For the sake of comparison, we also show the response to the compound stimulus presented as adapter in repetition trials (green line). Because of the similarity of the results obtained in each monkey separately (Fig. $5 B, C$ ), we pooled the data of the two animals in Figure $5 A$. As predicted by both the ADNI and ADI models, repetition of the compound stimulus suppressed the response to the repeated compound stimulus (Fig. 5, compare green and red lines; twosided Wilcoxon matched-pairs test: $p<0.000001, N=81$ neurons), and this held true in each monkey (both $p$ values $<0.0005$ ).

In agreement with the second prediction of the ADNI model, the mean gross response to the compound stimulus following a single effective stimulus was smaller than that to the same compound stimulus when being repeated (Fig. 5, compare blue and red lines; two-sided Wilcoxon matched pairs test: $p<0.005, N=$ 81 neurons). Given that this effect changed during the course of the response, we tested the significance of differences in response between these two conditions over time. To do so, we first computed the responses for each of the two conditions in five nonoverlapping, successive $50 \mathrm{~ms}$ bins, with the first bin starting at 55 

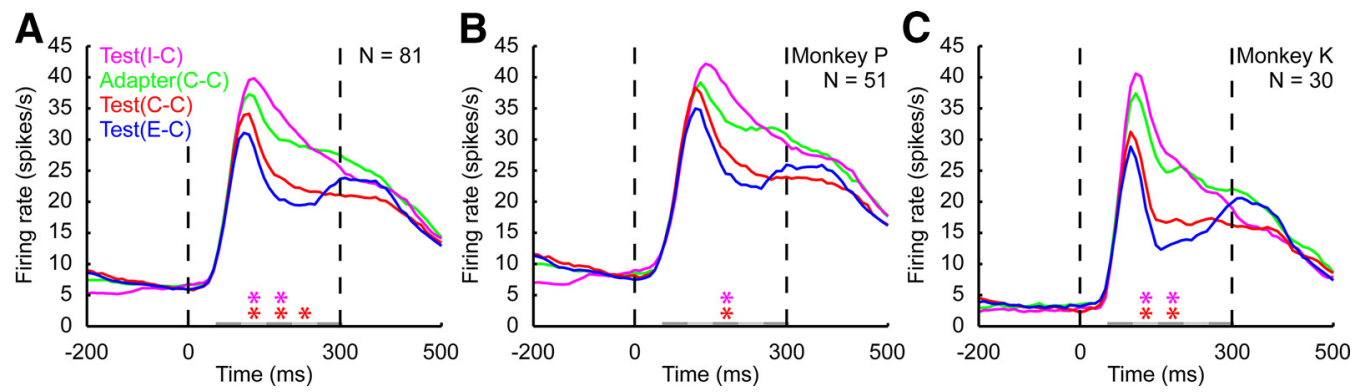

Figure 5. Effect of prior adaptation to the effective or ineffective stimulus on the response to the compound stimulus. Population peristimulus time histograms (PSTHs) of the responses to the compound stimulus presented as adapter (green) and test (red) in repetition trials as well as following either the effective (blue) or ineffective stimulus (magenta). $A-C$, The PSTHs were averaged across all responsive neurons of the two monkeys $(\boldsymbol{A})$ and of each monkey separately ( $\boldsymbol{B}$, Monkey $\mathrm{P} ; \boldsymbol{C}$, Monkey $\mathrm{K}$ ). Asterisks indicate significant differences in response to the compound stimulus when presented as adapter in repetition trials versus following the ineffective stimulus (magenta) and when being repeated versus following the effective stimulus (red). As indicated by the horizontal bars of alternating gray levels, the responses were compared in five successive $50 \mathrm{~ms}$ nonoverlapping time windows, with the first window starting at $55 \mathrm{~ms}$ after stimulus onset. To compare the responses, for each time window we applied a two-sided Wilcoxon matched-pairs test and then performed the FDR correction (Benjamini and Hochberg, 1995) to account for multiple comparisons (significance threshold $q<0.05$ ). Same conventions and selection of neurons as in Figure $2 A$.

ms after stimulus onset. We then applied a two-sided Wilcoxon matched-pairs test for each thus-defined time bin followed by a correction for multiple comparisons [false discovery rate (FDR): significance threshold $q<0.05, N=5$ comparisons; Benjamini and Hochberg, 1995]. There was a significantly larger response from 105 to $255 \mathrm{~ms}$ after stimulus onset to the repeated compound stimulus than to the same compound stimulus following the effective stimulus (Fig. 5A, red asterisks; $N=81$ neurons). The difference between these two conditions was significant in each monkey (Fig. $5 B, C$ ).

Last, we also confirmed the third prediction from the divisive normalization ADNI model; namely, a response enhancement to the compound stimulus following adaptation to the ineffective stimulus (Fig. 5, compare magenta and green lines; two-sided Wilcoxon matched-pairs test: $p<0.05, N=81$ neurons). Further testing using the $50 \mathrm{~ms}$ time bins showed that this response enhancement was significant from 105 to $205 \mathrm{~ms}$ after stimulus onset (Fig. 5A, magenta asterisks; FDR: $q<0.01$ ) and present in each monkey (Fig. $5 B, C$ ). This response enhancement was not due to the incapacity of the ineffective stimulus to suppress responses, as evidenced by the observation of a suppressed response to the repeated ineffective stimulus (see Repetition suppression; Fig. 3B).

The changes in response to the compound stimulus due to adaptation to a single (effective or ineffective) stimulus that were predicted by the divisive normalization ADNI model took place only in the first part of the response (Fig. 5). Remarkably, the later part of the response showed a reversal of the sign of the adaptation effects compared with the first part of the response. Such reversal of the adaptation effects was observed in each monkey (Fig. 5B,C). Thus, the ADNI model appears to capture well the initial but not the late part of the response of IT cortex neurons.

\section{Fitting responses of neurons by divisive normalization models}

We fitted the ADNI model (Eq. 1) that included adaptation of both the driving and normalizing inputs to the neural responses of each individual position $\times$ neuron combination. For the sake of comparison, we also fitted a divisive normalization model that allowed adaptation of the driving input, but not the divisive input (the ADI model; Eq. 2). The model fitting was performed for the gross responses obtained in a $100 \mathrm{~ms}$ window that started $105 \mathrm{~ms}$ after stimulus onset, i.e., the strongest phase of the response for which sizable adaptation effects were observed. Both models fit- ted overall the wide range of the responses in the different stimulus conditions (e.g., responses to the effective and ineffective stimuli presented as adapters and tests) rather well, with a mean $r^{2}$ of 0.93 (SD, 0.09) and 0.90 (SD, 0.12) for the ADNI and ADI model, respectively. However, despite the equal number of free parameters in the two models, the ADNI model significantly outperformed the ADI model (two-sided Wilcoxon matched-pairs test: $p<0.000001, N=151$ position $\times$ neuron combinations, $N=81$ neurons). Similar results were obtained when analyzing the data of each monkey separately (Fig. 6E; two-sided Wilcoxon matched-pairs test: both $p$ values $<0.0005)$. The differences in fits between the two models can be appreciated when comparing the mean observed and predicted responses for the four compound stimulus conditions (Fig. $6 A, B$ ). The ADNI model fitted both the enhancement of the response to the compound stimulus following the ineffective stimulus and the stronger suppression of the response to the compound when it followed the effective stimulus compared with the repeated compound stimulus (Fig. 6A). Importantly, the ADI model did not predict these two observed effects, producing equal average responses to the compound stimulus presented as adapter and when following the ineffective stimulus as well as equal responses to the compound stimulus following the effective stimulus and the repeated compound stimulus (Fig. 6B). The better fits of the ADNI compared with the ADI model held true regardless of whether the ineffective stimulus was excitatory or inhibitory when presented alone. To show this, we split up the position $\times$ neuron combinations into three groups, depending on their net response to the ineffective stimulus presented as adapter in repetition trials: inhibitory response (net response $\leq-2.5$ spikes/s), no response $(-2.5$ spikes/s $<$ net response $\leq 2.5$ spikes $/ \mathrm{s}$ ) and excitatory response (net response $>2.5$ spikes $/ \mathrm{s}$ ). The net responses were computed using a $300 \mathrm{~ms}$ analysis window starting at $50 \mathrm{~ms}$ after stimulus onset and a baseline window of 100 $\mathrm{ms}$ before stimulus onset. We found that for each of these three groups of position $\times$ neuron combinations, the average fit of the ADNI model was significantly higher than that obtained with the ADI model (two-sided Wilcoxon matched-pairs test: all $p$ values $<0.01$ ). This can also be appreciated in Figure $6 C, D$, which plots the predicted contrast indices (I3) computed for the compound stimulus following adaptation to the ineffective stimulus versus the compound stimulus presented as adapter against the observed indices for each fitted position $\times$ neuron combination. The index $I 3$ was computed as follows: $I 3=[\operatorname{resp}(I-C)-\operatorname{resp}(C)] /[\operatorname{resp}(I-C)+\operatorname{resp}(C)]$, 

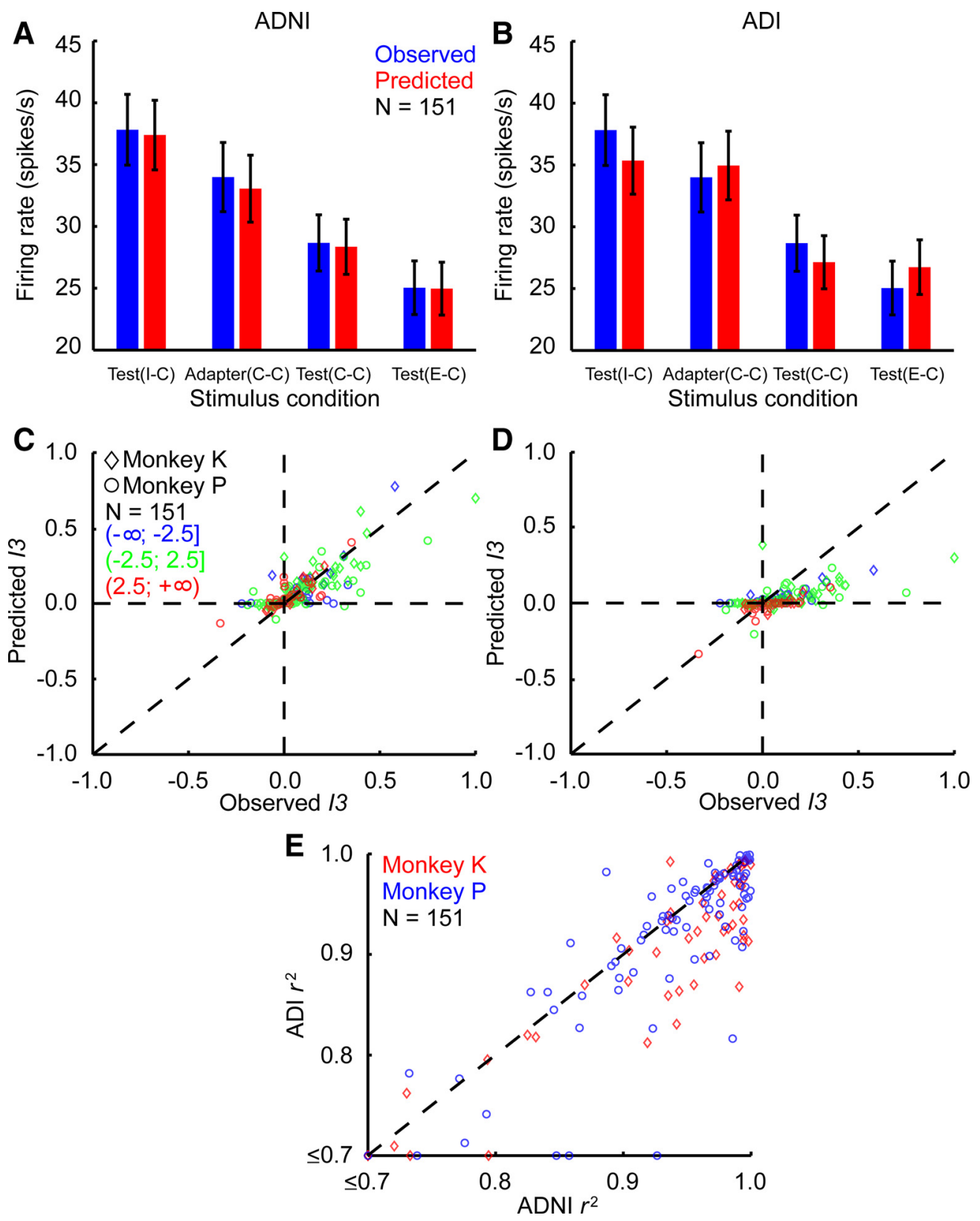

Figure 6. Observed and predicted responses compared. Both models incorporate driving and divisive normalizing inputs as well as adaptation of the driving input, with only the ADNI model (and not the ADI model) also allowing adaptation of the divisive normalizing input. $\boldsymbol{A}, \boldsymbol{B}$, Mean observed (blue) and predicted (red) responses to the compound stimulus presented as adapter $[$ Adapter $(C-C)]$ and test $[$ Test $(C-C)]$ in repetition trials as well as following either the effective $[$ Test $(E-C)]$ or ineffective $[$ Test $(I-C)]$ stimulus. Bars indicate $S E s$ of the mean. $C, D, 0 b s e r v e d$ versus predicted $/ 3$ indices for both the ADNI and ADI models. 13 measures the response facilitation for the compound stimulus due to adaptation to the ineffective stimulus, with higher positive values indicating a stronger response facilitation. Each point corresponds to a single position $\times$ neuron combination. Data of the two animals were pooled and indicated by different symbols. Different colors correspond to the three different selections of position $\times$ neuron combinations defined on the basis of the net firing rate for the ineffective stimulus presented as adapter in repetition trials. The first group (blue symbols) included only those position $\times$ neuron combinations for which the net response to the ineffective stimulus was $<-2.5$ spikes $/ s$, whereas the second (green symbols) and third (red symbols) groups comprised the position $\times$ neuron combinations with the net response to the ineffective stimulus being between -2.5 and 2.5 spikes $/ \mathrm{s}$ and $>2.5$ spikes $/ \mathrm{s}$, respectively. $E$, Comparison of the goodness of fit between the ADNI and ADI model. Each symbol (blue circles, Monkey P; red diamonds, Monkey K) corresponds to a single analyzed position $X$ neuron combination, with the abscissa and the ordinate being the $r^{2}$ of the ADNI and ADI model, respectively. Each panel is based on the same set of position $\times$ neuron combinations, with $N$ denoting their number.

where $\operatorname{res} p(X)$ denotes the (predicted or observed) response to stimulus $X$, and $I$ - $C$ and $C$ correspond to the responses to the compound stimulus following the ineffective stimulus $(I)$ and when presented as adapter, respectively. The index $I 3$ measures the response facilitation for the compound stimulus due to adaptation to the ineffective stimulus, with higher positive values indicating a stronger response facilitation. It is obvious that the ADNI model (Fig. $6 \mathrm{C}$ ) fitted the response enhancement (positive contrast indices) for the compound stimulus following the ineffective stimulus better than the ADI model (Fig. $6 D$ ) for each of the three groups of position $\times$ neuron combinations. Even when the ineffective stimulus was inhibitory (blue symbols), the ADI model strongly underestimated the observed response enhancement.

The median (quartiles) fitted values of the ADNI model were $0.49(0.30-0.76)$ and $0.40(0.04-0.86)$ for the adaptation parameters $\beta$ and $\gamma$, respectively. Noteworthy was the large interquartile 
range $(0.82)$ for the adaptation parameter $\gamma$ of the divisive normalization term, indicating large between-neuron heterogeneity with respect to the suppression of the divisive normalization term. Nonetheless, the Spearman rank correlation between $\beta$ and $\gamma$ was $0.63(p<0.05, N=151$ position $\times$ neuron combinations $)$, indicating that the degree of the adaptation-induced suppression correlated between the driving and divisive inputs.

The median (quartiles) fitted value of the tuned normalization parameter $\alpha$ was $0.89(0.23-2.32)$ for the ADNI model, which was less than expected from averaging of the effective and ineffective inputs $(\alpha=1)$. The normalization parameter $\alpha$ was significantly correlated with the facilitation contrast index $I 3$ computed using the predicted responses of the ADNI model (Spearman rank correlation: $r=0.49, p<0.000001$ ). This agrees with the notion that neurons for which the ineffective stimulus is more effective in suppressing the response to the compound stimulus also show a more enhanced response to the compound stimulus following adaptation to the ineffective stimulus. Thus, one would predict that the response facilitation observed in a neuron depends on the degree of the suppressive effect generated by the ineffective stimulus in that neuron. To verify this prediction, we correlated the $I 2$ (see above) and $I 3$ indices, computed for actual observed neural responses. Recall that the index $I 2$ compares the response to the compound stimulus with the response to the effective stimulus when presented alone. Hence, higher values of index $I 2$ correspond to a stronger suppressive effect of the ineffective stimulus. The $I 2$ and $I 3$ indices were computed on independent sets of half of the trials to avoid spurious correlations due to the presence of an identical condition in the two indices (compound stimulus presented as adapter). In agreement with the prediction, the correlation between the observed $I 2$ and $I 3$ indices was positive and significant (Spearman rank correlation: $I 2$ odd trials, $I 3$ even trials: $r=0.22, p<0.005$; $I 2$ even trials, $I 3$ odd trials: $r=0.31, p<0.0001$; in both cases, $N=151$ position $\times$ neuron combinations).

The ADNI and ADI models were tuned divisive normalization models (Ni et al., 2012). We also fitted less general divisive normalization models in which the degree of normalization was equal for the effective and ineffective inputs, with $\alpha$ being a free parameter that equally affected both the effective and ineffective inputs. The untuned divisive normalization version of the ADNI model performed significantly worse [two-sided Wilcoxon matched-pairs test: $p<0.00005$; mean (SD) $r^{2}=0.90(0.12)$, $N=151$ position $\times$ neuron combinations, $N=81$ neurons] than the tuned normalization model, despite an equal number of free parameters. The untuned ADI model also performed worse $\left[r^{2}=\right.$ $0.88(0.14)]$ than its tuned version, but this difference did not reach significance. Despite the worse fits, both untuned models made qualitatively the same predictions as their tuned versions, with the response enhancement for the compound stimulus following adaptation to the ineffective stimulus being present for the ADNI model. Furthermore, similar to the tuned normalization models, the untuned ADNI model fitted the data better than the untuned ADI model (two-sided Wilcoxon matched-pairs test: $p<0.0005, N=151$ position $\times$ neuron combinations).

We also modeled the responses of a neuron as a linear sum of the direct bottom-up inputs due to presentation of the effective (Eff) and ineffective (InEff) stimuli. Similar to the ADI model, we allowed a differential adaptation of each input in this "linear" model by using two independent adaptation parameters $\beta$ and $\mu$. Like in the ADNI and ADI models, we included a parameter $\alpha$ that reflected the degree of contribution of the ineffective stimulus relative to the effective stimulus to the total response of a neuron. Thus, the linear model included the same number of free parameters (Eff, InEff, $\beta, \mu, \alpha$ ) as the ADNI and ADI models (see Materials and Methods). To fit the linear model, we used the same parameters, constraints, and fitting procedure as those used to fit the ADNI and ADI models. We observed a significantly worse goodness of fit of the linear model [mean (SD) $r^{2}$ $=0.75(0.23), N=151$ position $\times$ neuron combinations] compared with those of the tuned ADNI and ADI models (two-sided Wilcoxon matched-pairs test: both $p$ values $<$ $0.000001)$. The latter also held true when analyzing the data of each monkey separately.

\section{Eye-movement analysis}

Analysis of the eye movements of each monkey showed that none of the reported effects in spiking activity can be attributed to the miniature differences in eye position between the stimulus conditions. Mean eye positions between the upper single-stimulus presentations, lower single-stimulus presentations, and the compound stimuli, with all being presented as adapter, differed by $<0.06^{\circ}$. The latter was true for both the horizontal and vertical dimensions. These are negligible differences in eye position given the relatively large size of receptive fields of anterior IT cortex neurons (Op De Beeck and Vogels, 2000). The same held true for the eye positions measured during the compound stimulus presented as test, sorted according to whether the preceding adapter was present in the upper visual field, lower visual field, or was the same compound stimulus (Fig. 7). Monkey K showed a remarkable pattern in eye movements $>200 \mathrm{~ms}$ after stimulus onset. Specifically, there was a small $\left(<0.1^{\circ}\right)$ shift toward the position of the stimulus that differed from the position of the adapter. The eye movements of the other animal (Monkey P) for the compound stimulus presented as test also seemingly depended on the adapter condition. However, this dependency is unconvincing given its presence already at stimulus onset. Importantly, the small but consistent difference in eye movements observed in Monkey $\mathrm{K}$ cannot explain the response enhancement for the compound stimulus following the ineffective stimulus because the response enhancement occurred earlier than these changes in eye position.

\section{Discussion}

Here we report three novel findings predicted by a divisive normalization model incorporating adaptation of both the direct and divisive inputs. First, IT cortex neurons show repetition suppression for compound stimuli that consisted of the simultaneous presentation of both effective and ineffective stimuli. Second, adaptation of IT cortex neurons to an effective stimulus produces a stronger suppression of the initial part of the response to the compound stimulus than adaptation to the same compound. Third and most importantly, adaptation to an ineffective stimulus enhances the initial part of the response of IT cortex neurons to the compound stimulus. These findings indicate that both the adapter and test stimulus configuration determines whether adaptation suppresses or enhances the responses of IT cortex neurons. The stimulus-dependent adaptation effects observed in the initial part of the response could be well fitted by divisive normalization models in which both the driving and divisive normalizing inputs are suppressed by adaptation but not by a model that allows adaptation of only the driving but not the divisive normalizing inputs.

Spatial attention can bias responses of IT cortex neurons to the compound stimulus. Specifically, attention to an effective stimulus part of the compound stimulus is expected to increase 

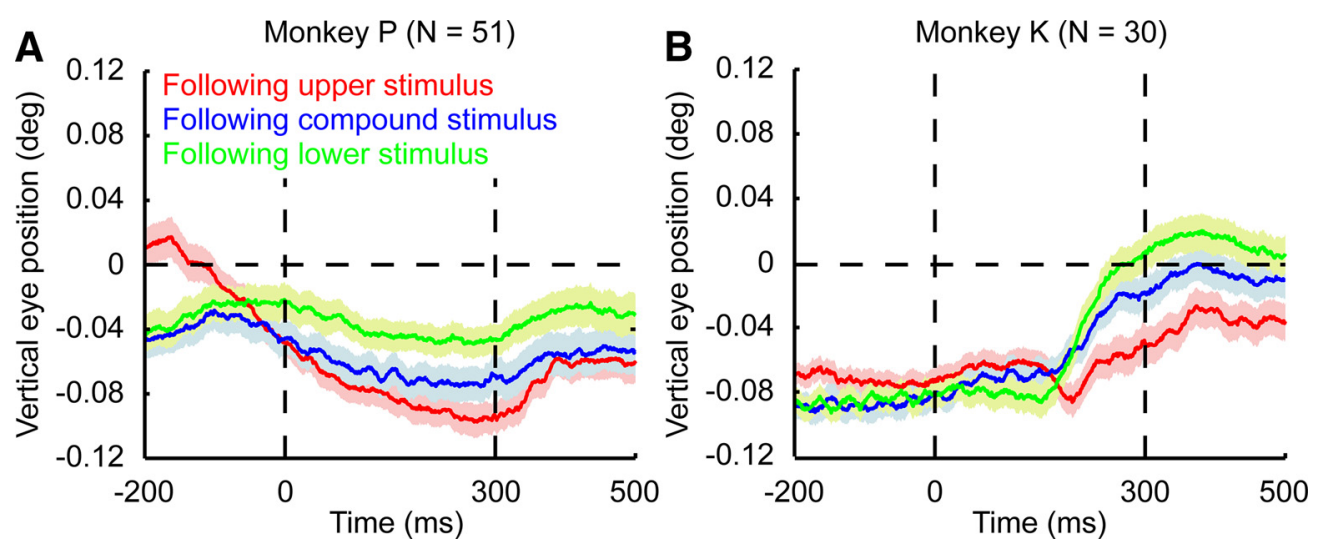

Figure 7. Effect of the position of adapter stimulus on eye movements for the compound stimulus presented as test. $A, B$, Mean baseline-corrected vertical eye position for the compound stimulus presented as test when following adapter stimulus presentation either in the upper (above the fixation target; red) or lower (below the fixation target; green) visual field as well as when being repeated (blue). For each analyzed neuron and position, we first averaged the mean eye-position signals for the compound stimulus across both the effective and ineffective stimuli (see Materials and Methods). In case of the repeated compound stimulus, the eye-position signals were averaged across the two relative positions of the individual images. Then the thus-derived mean eye-position signals per neuron were averaged across all analyzed neurons in each monkey separately. For each monkey ( $\boldsymbol{A}$, Monkey $\mathrm{P} ; \boldsymbol{B}$, Monkey $\mathrm{K}$ ) we used the same selection of neurons as that used in the analyses of spiking activity. Stippled vertical lines indicate onset and offset of the compound stimulus presented as test, whereas bands indicate SEM. $N$ denotes the number of analyzed neurons.

the response to that compound stimulus compared with when attention is diverted away from it (Chelazzi et al., 1998). Conversely, attention to the ineffective constituent of the compound stimulus is expected to decrease the response to the compound stimulus (Chelazzi et al., 1998). One may hypothesize that the adapter stimulus biased attention toward that stimulus when it was presented as a part of the compound stimulus. If so, the response to the compound stimulus following the ineffective stimulus is expected to be suppressed and, conversely, enhanced when following the effective stimulus. However, this was the opposite of what we have observed for the initial part of the response of IT cortex neurons, thus falsifying such attention scheme as a valid explanation of the present results. This may not be surprising given that the cue-based attention effects are known to occur $\sim 170$ ms after stimulus onset (Chelazzi et al., 1998), which is after the mean response enhancement for the compound stimulus following the ineffective stimulus as observed in the present study.

The effects predicted by adaptation of the divisive normalization circuit were only present in the early part of the response. The smaller later part of the response showed a reversal of the adaptation effects compared with the early part. A time dependency of adaptation effects in the IT cortex have been reported before for repetitions of single stimuli (Liu et al., 2009; Kaliukhovich and Vogels, 2012). However, the origin of the time dependency of adaptation effects for single stimuli in the IT cortex still remains unclear, with potential candidates being recurrent processing within the IT cortex or top-down feedback from higher areas. These late processes may also underlie the reversal of the adaptation effects for the compound stimuli reported in the present study. Stimulus interaction effects in the IT cortex are known to occur early during the course of the response (Chelazzi et al., 1998; Missal et al., 1999), whereas the top-down modulations operate and thus can influence such interactions later in time (Chelazzi et al., 1998). This agrees well with the adaptationrelated normalization effects being only present in the early part of the response. Hence, in the later part of the response other factors beyond adaptation can affect the divisive normalization circuit, thus masking or even disrupting the changes in divisive normalization due to adaptation.
The response to the ineffective stimulus, averaged across our sample of neurons, was rather weak and short (Fig. 2A). Nonetheless, this small response was sufficient to produce repetition suppression (see Results). In contrast, adaptation to the weak ineffective stimulus enhanced the response to the compound stimulus. Thus, the same adapter stimulus had opposite effects, depending on the test stimulus: suppression of the response to the same (repeated) stimulus or enhancement of the response to the compound stimulus. Adaptation to the effective stimulus, on the other hand, suppressed the response to both the repeated effective stimulus and the compound stimulus. These different adaptation effects for the compound stimulus presented as test can be well explained by an adaptation-induced decrease of not only the direct input to a neuron but also of the divisive normalizing input, as has been demonstrated by our simulations (Fig. 4) and fitting such a model to the responses of individual neurons (Fig. 6). The biophysical mechanisms behind normalization in the IT cortex are still poorly understood, as in other visual cortical areas (Carandini and Heeger, 2012). A likely mechanism of divisive normalization for compound stimuli in the IT cortex is inhibition. GABAergic inhibition has been demonstrated to play an important role in shaping the stimulus selectivity of single IT cortex neurons (Wang et al., 2000), but its role in the shape interactions studied here remains to be assessed. One possible candidate of such a framework is a network of interconnected neurons with different feature preferences that inhibit each other (Moldakarimov et al., 2005). In such a network, adaptation of the neurons that prefer the features present in the adapter stimulus will reduce their response and, as a result, their inhibitory impact (through GABAergic interneurons) on the neurons that prefer the features of the nonadapted part of the compound stimulus. Such adaptation-induced reduced inhibition has also been postulated to underlie adaptation effects in V1 for large stimuli that encroach the surround of the receptive field (Solomon and Kohn, 2014). Altogether, this suggests that adaptation affects the balance between excitatory and inhibitory inputs to a neuron, and thus the outcome of adaptation will depend on how both the adapter and test stimuli will recruit these inputs. 
Normalization has been documented to operate at the different stages of the ventral visual pathway, including areas V1, V2, and V4 (Reynolds et al., 1999; Carandini and Heeger, 2012). As in other studies (Ni et al., 2012), our model assumes that the normalization occurs within the area of study. Given that we matched the stimuli and their spatial separation to IT cortex receptive fields, which are larger compared with the receptive fields of neurons in earlier visual areas, we believe that the interaction effects reported here largely originated within the IT cortex. Indeed, since the effective and ineffective stimuli differed in shape and color and were presented in different visual field quadrants, interactions in previous stages, such as V4, would have been mainly limited to center-surround interactions, with such interactions varying from enhancement to suppression of the response, depending on the differences in stimulus features (Schein and Desimone, 1990). Furthermore, the correlation between the strength of the stimulus interaction of a single IT cortex neuron and the response enhancement of that neuron following adaptation to the ineffective stimulus supports our belief that the normalization phenomenon we studied here occurs predominantly at the level of IT cortex neurons. Our model assumes that the inputs to the neurons become suppressed following adaptation but is agnostic about where the suppression occurs, i.e., before or within the IT cortex. Previous studies showed that adaptation to single stimuli in the IT cortex tolerates changes in the location of the adapter and test stimuli (Lueschow et al., 1994; De Baene and Vogels, 2010), thus suggesting that at least part of the repetition suppression is not inherited from earlier stages, such as V1 or V4.

The response enhancement for compound stimuli following an ineffective stimulus and the response suppression for compound stimuli that follow the effective stimulus implies that the response of neurons that signal the novel stimulus within the compound configuration will be enhanced while the response of neurons that signal the recently seen stimulus will be reduced. This results in an increase of the saliency of the novel stimulus within the configuration (or a reduction of the saliency of the recently seen stimulus). This adaptation-induced change in stimulus saliency may explain the bias toward the novel stimulus location present in the late part of the eye movements for the compound stimulus presented as test in Monkey K (Fig. 7). In fact, such an eye position bias toward the "novel" stimulus location has been observed in several human studies (Watanabe, 2001). Furthermore, the adaptation-induced change in saliency may underlie the faster and enhanced detection of a peripheral stimulus presented at a different location than a preceding uninformative cue (Posner and Cohen, 1984). The cause of this socalled "inhibition of return" effect is still debated (Hilchey et al., 2014; Zhao and Heinke, 2014), but, in agreement with our findings, sensory adaptation may be the cause of at least part of this effect (Posner and Cohen, 1984; Patel et al., 2010; Satel et al., 2011).

The present and other studies (Kaliukhovich et al., 2013) of the IT and early visual cortex (Solomon and Kohn, 2014) suggest that one role of adaptation is to decrease the saliency of recently seen stimuli. If so, adaptation thus serves to increase the processing of stimuli that differ from recently seen ones, even when these occur within a configuration of multiple stimuli.

\section{References}

Bar M, Biederman I (1999) Localizing the cortical region mediating visual awareness of object identity. Proc Natl Acad Sci U S A 96:1790-1793. CrossRef Medline
Benjamini Y, Hochberg Y (1995) Controlling the false discovery rate: a practical and powerful approach to multiple testing. J Roy Statist Soc Ser B 57:289-300.

Carandini M, Heeger DJ (2012) Normalization as a canonical neural computation. Nat Rev Neurosci 13:51-62. CrossRef Medline

Chelazzi L, Duncan J, Miller EK, Desimone R (1998) Responses of neurons in inferior temporal cortex during memory-guided visual research. J Neurophysiol 80:2918-2940. Medline

Clifford CW, Webster MA, Stanley GB, Stocker AA, Kohn A, Sharpee TO, Schwartz O (2007) Visual adaptation: neural, psychological and computational aspects. Vision Res 47:3125-3131. CrossRef Medline

De Baene W, Vogels R (2010) Effects of adaptation on the stimulus selectivity of macaque inferior temporal spiking activity and local field potentials. Cereb Cortex 20:2145-2165. CrossRef Medline

De Baene W, Premereur E, Vogels R (2007) Properties of shape tuning of macaque inferior temporal neurons examined using rapid serial visual presentation. J Neurophysiol 97:2900-2916. CrossRef Medline

Dekel R, Sagi D (2015) Tilt aftereffect due to adaptation to natural stimuli. Vision Res 117:91-99. CrossRef Medline

Desimone R (1996) Neural mechanisms for visual memory and their role in attention. Proc Natl Acad Sci U S A 93:13494-13499. CrossRef Medline

Dhruv NT, Tailby C, Sokol SH, Lennie P (2011) Multiple adaptable mechanisms early in the primate visual pathway. J Neurosci 31:15016-15025. CrossRef Medline

Ewbank MP, Rhodes G, von dem Hagen EA, Powell TE, Bright N, Stoyanova RS, Baron-Cohen S, Calder AJ (2015) Repetition suppression in ventral visual cortex is diminished as a function of increasing autistic traits. Cereb Cortex 25:3381-3393. CrossRef Medline

Hilchey MD, Klein RM, Satel J (2014) Returning to "inhibition of return" by dissociating long-term oculomotor IOR from short-term sensory adaptation and other nonoculomotor "inhibitory" cueing effects. J Exp Psychol Hum Percept Perform 40:1603-1616. CrossRef Medline

Kaliukhovich DA, Vogels R (2011) Stimulus repetition probability does not affect repetition suppression in macaque inferior temporal cortex. Cereb Cortex 21:1547-1558. CrossRef Medline

Kaliukhovich DA, Vogels R (2012) Stimulus repetition affects both strength and synchrony of macaque inferior temporal cortical activity. J Neurophysiol 107:3509-3527. CrossRef Medline

Kaliukhovich DA, Vogels R (2014) Neurons in macaque inferior temporal cortex show no surprise response to deviants in visual oddball sequences. J Neurosci 34:12801-12815. CrossRef Medline

Kaliukhovich DA, De Baene W, Vogels R (2013) Effect of adaptation on object representation accuracy in macaque inferior temporal cortex. J Cogn Neurosci 25:777-789. CrossRef Medline

Liu Y, Murray SO, Jagadeesh B (2009) Time course and stimulus dependence of repetition-induced response suppression in inferotemporal cortex. J Neurophysiol 101:418-436. CrossRef Medline

Lueschow A, Miller EK, Desimone R (1994) Inferior temporal mechanisms for invariant object recognition. Cereb Cortex 4:523-531. CrossRef Medline

Malach R (2012) Targeting the functional properties of cortical neurons using fMR-adaptation. Neuroimage 62:1163-1169. CrossRef Medline

McMahon DB, Olson CR (2007) Repetition suppression in monkey inferotemporal cortex: relation to behavioral priming. J Neurophysiol 97: 3532-3543. CrossRef Medline

Miller EK, Li L, Desimone R (1991) A neural mechanism for working and recognition memory in inferior temporal cortex. Science 254:1377-1379. CrossRef Medline

Miller EK, Gochin PM, Gross CG (1993) Suppression of visual responses of neurons in inferior temporal cortex of the awake macaque by addition of a second stimulus. Brain Res 616:25-29. CrossRef Medline

Missal M, Vogels R, Li CY, Orban GA (1999) Shape interactions in macaque inferior temporal neurons. J Neurophysiol 82:131-142. Medline

Moldakarimov S, Rollenhagen JE, Olson CR, Chow CC (2005) Competitive dynamics in cortical responses to visual stimuli. J Neurophysiol 94:33883396. CrossRef Medline

Müller K, Schillinger F, Do DH, Leopold DA (2009) Dissociable perceptual effects of visual adaptation. PLoS One 4:e6183. CrossRef Medline

Ni AM, Ray S, Maunsell JH (2012) Tuned normalization explains the size of attention modulations. Neuron 73:803-813. CrossRef Medline

Noudoost B, Esteky H (2013) Neuronal correlates of view representation 
revealed by face-view aftereffect. J Neurosci 33:5761-5772. CrossRef Medline

Ohshiro T, Angelaki DE, DeAngelis GC (2011) A normalization model of multisensory integration. Nat Neurosci 14:775-782. CrossRef Medline

Op De Beeck H, Vogels R (2000) Spatial sensitivity of macaque inferior temporal neurons. J Comp Neurol 426:505-518. CrossRef Medline

Patel SS, Peng X, Sereno AB (2010) Shape effects on reflexive spatial selective attention and a plausible neurophysiological model. Vision Res 50: 1235-1248. CrossRef Medline

Patterson CA, Wissig SC, Kohn A (2013) Distinct effects of brief and prolonged adaptation on orientation tuning in primary visual cortex. J Neurosci 33:532-543. CrossRef Medline

Posner MI, Cohen Y (1984) Components of visual orienting. In: Attention and performance $\mathrm{X}$ : control of language processes (Bouma $\mathrm{H}$, Bonwhuis D, eds), pp 551-556. Hillsdale, NJ: Lawrence Erlbaum Associates.

Reynolds JH, Heeger DJ (2009) The normalization model of attention. Neuron 61:168-185. CrossRef Medline

Reynolds JH, Chelazzi L, Desimone R (1999) Competitive mechanisms subserve attention in macaque areas V2 and V4. J Neurosci 19:1736-1753. Medline

Richmond BJ, Wurtz RH, Sato T (1983) Visual responses of inferior temporal neurons in awake rhesus monkey. J Neurophysiol 50:1415-1432. Medline

Rolls ET, Tovee MJ (1995) The responses of single neurons in the temporal visual cortical areas of the macaque when more than one stimulus is present in the receptive field. Exp Brain Res 103:409-420. Medline

Satel J, Wang Z, Trappenberg TP, Klein RM (2011) Modeling inhibition of return as short-term depression of early sensory input to the superior colliculus. Vision Res 51:987-996. CrossRef Medline

Sato T (1989) Interactions of visual stimuli in the receptive fields of inferior temporal neurons in awake macaques. Exp Brain Res 77:23-30. Medline

Sawamura H, Orban GA, Vogels R (2006) Selectivity of neuronal adaptation does not match response selectivity: a single-cell study of the fMRI adaptation paradigm. Neuron 49:307-318. CrossRef Medline

Schein SJ, Desimone R (1990) Spectral properties of V4 neurons in the macaque. J Neurosci 10:3369-3389. Medline

Solomon SG, Kohn A (2014) Moving sensory adaptation beyond suppressive effects in single neurons. Curr Biol 24:R1012-R1022. CrossRef Medline

Vogels R, Sáry G, Orban GA (1995) How task-related are the responses of inferior temporal neurons? Vis Neurosci 12:207-214. CrossRef Medline

Wang Y, Fujita I, Murayama Y (2000) Neuronal mechanisms of selectivity for object features revealed by blocking inhibition in inferotemporal cortex. Nat Neurosci 3:807-813. CrossRef Medline

Watanabe K (2001) Inhibition of return in averaging saccades. Exp Brain Res 138:330-342. Medline

Wissig SC, Kohn A (2012) The influence of surround suppression on adaptation effects in primary visual cortex. J Neurophysiol 107:3370-3384. CrossRef Medline

Zhao Y, Heinke D (2014) What causes IOR? Attention or perception?Manipulating cue and target luminance in either blocked or mixed condition. Vision Res 105:37-46. CrossRef Medline

Zoccolan D, Cox DD, DiCarlo JJ (2005) Multiple object response normalization in monkey inferotemporal cortex. J Neurosci 25:8150-8164. CrossRef Medline 